\title{
RSS-Distance Rationalization Procedure for Localization in an Indoor Environment
}

\author{
Adeniran Ademuwagun \\ University of Aberdeen King's College, Aberdeen, UK \\ Email: r01aka13@abdn.ac.uk
}

How to cite this paper: Ademuwagun, A. (2019) RSS-Distance Rationalization Procedure for Localization in an Indoor Environment. Wireless Sensor Network, 11, 13-33.

https://doi.org/10.4236/wsn.2019.112002

Received: January 18, 2019

Accepted: February 25, 2019

Published: February 28, 2019

Copyright $\odot 2019$ by author(s) and Scientific Research Publishing Inc. This work is licensed under the Creative Commons Attribution International License (CC BY 4.0).

http://creativecommons.org/licenses/by/4.0/

(c) (i) Open Access

\begin{abstract}
The computational capabilities of off-the-shelf wireless sensors networks presents a limitation when more complex forms of localization algorithms are employed for location estimation purposes, particularly in an indoor environment. Range-free algorithms rely on Received Signal Strength (RSS) from sensors that are location aware (anchor nodes) as the major means of distance estimation. This paper presents a non-site specific algorithm for better estimating RSS relationship with distance. By employing a unique form of rationalization of raw RSS with respect to distance using the proposed algorithm, it is possible to enhance the reliability of RSS when employed in indoor Localization Algorithms. Consequently, this paper presents an innovative RSS-Distance rationalization algorithm for localization of objects in an indoor environment. The paper compared the proposed algorithm with Simple Moving Average (SMA) algorithm due to the wide applicability and ease of manipulation of SMA. The analysis of the proposed algorithm and SMA shows that the proposed algorithm better modifies RSS for more accurate position estimation in an indoor environment.
\end{abstract}

\section{Keywords}

Anchor Nodes, Received Signal Strength (RSS), Localization

Algorithm, Simple Moving Average (SMA)

\section{Introduction}

Localization using wireless sensors relies on inter-nodal distance measurements between anchors using the coordinate system or the knowledge of the enclosed geographical environment. The measurements, using a typical WSN localization system can be related to the coordinates of sensors using the following generic formula: 


$$
\boldsymbol{S}=\boldsymbol{h}(\boldsymbol{U})+\boldsymbol{e}
$$

where $S$ is the vector of all the measurements, $U$ contains the true coordinate vectors of sensors whose locations are to be estimated and $\boldsymbol{e}$ is the vector due to measurement errors [1]. For most applications, it is pointless to sense data without the awareness of the nodes position or location. It is only when sensor nodes are rightly sited, that the events of interest can be appropriately monitored [2] [3] [4].

RSS is a free resource that is exploited by both range-based and range-free algorithms, however, range-based algorithms are more accurate but they do require a higher computational cost and complexity than range free algorithm. WSN for range-based algorithms would require modification and consequently be more expensive than WSN devices for range-free algorithms [5] [6]. Off-the-shelf sensor nodes can be used to implement range-free algorithms, hence our interest in RSS as a free resource for localization algorithms.

The simplest form of measurement in WSN is connectivity measurements [7]. In this type of measurement, sensors receive signals from other sensors within its transmission range. These signals can be translated into binary distance measurements, which implies that it is either a sensor which is within range or not. Most range-free localization algorithms such as Centroid and DV-Hop localization algorithms, which are proximity based algorithms, depend largely on reliable RSS quality and not its direct relationship with distance for accurate position estimation. Nevertheless, it becomes essential to develop a procedure for establishing a reliable RSS-distance relationship, for more accurate positioning localization algorithms. In this paper, we propose a unique procedure for modifying the RSS data from sensor nodes for reliable distance estimation.

The objective of this paper is to establish a relationship between RSS and distance. The paper will be developed in two phases. The first phase will present a justification through experimentation that RSS is a reliable indicator or factor for localization. The second phase will present the RSS-distance normalization algorithm. Thus, the goal is to present an algorithm for processing RSS values towards a 2D node localization scheme in an indoor environment. Since RSS values are used for approximating the spatial position of the sensor nodes, hence, our interest in this method. This is because sensor nodes are freely equipped with RF modules for wireless communication, thus, no extra hardware will be required. The paper is organized as follows; a synopsis of related literature and related theory, a description of the experimental test-bed, RSS as a reliable connectivity index; RSS-distance normalization algorithm, making a comparative analysis with Simple Moving Average (SMA); discussion and findings, and lastly, concluding the paper.

\section{Related Literature}

From literature study of indoor localization, it is established that most approaches to localization rely on a form of RSS for offline training using a priori 
fingerprinting information corresponding to the reference nodes. Some methods such as Maximum Likelihood Estimate (MLE) are also well documented [8] and have made significant contributions to localization research.

In this particular work, the Cramer-Rao Lower bound and MLE were derived for algorithms based on TOA and RSS. This requires that all devices in the network provide estimated range between themselves and their neighboring reference nodes. Localization accuracy is largely dependent on anchor or node densities. Hence, accuracy could become an issue in low density networks. On the other hand, large data overhead could negatively impact data transmission rate and life span of the network.

The effects of antenna polarization on localization accuracy of objects in an indoor environment were examined. The research established that the accuracy of localization was significantly related to the position of range measurement [9]. Also, it was established that the accuracy of localization is significantly related to the precision of range measurement. The research attempted to show that polarization angle affect RSS values and consequently the final localization estimate.

Various forms of weighted algorithms were also proposed. Basically, a rational attenuation exponent was developed, then the position of the targeted node is established through a form of centroid localization [1]-[8] [10]-[17]. Other concepts divided anchors into different groups and each group with a Path Loss Exponent (PLE), or the PLE is iteratively estimated from the anchor node of interest and based on the self-estimated PLE, calculate its own position [11] [12].

Some RSS-based location estimation algorithm for WSN combines Generalized Regression Neural Network (GRNN) and weighted centroid localization (WCL). To mitigate the instability of RSS, they proposed real-time training scheme that was capable of adapting to the changes in channel characteristics of WSN. They opined that the scheme was flexible and could match the spatial and temporal changes in wireless channel environment. In the algorithm, reference sensor nodes were permanently installed in some known locations. RSS measurements at all the access points (AP) from the reference sensors and their known locations (two dimensional coordinate data) were separately used to train two GRNN networks separately. After estimating the approximate location of the unknown node and its neighbors using GRNN, the final estimation is done by WCL [15].

Although existing literature review on the reliability of RSS is inexhaustible, nevertheless, there is a general agreement that raw RSS values in an indoor environment are unreliable with respect to distance. Also, what is clear from the foregoing is that most algorithms do rely on RSS in one form or another for finger printing or terrain mapping to estimate location. However, to the best of our knowledge there is no proposal to directly alter the reliability of RSS with respect to distance, prior to its direct application to localization. Consequently, our proposed algorithm seeks to fill this gap. 


\section{Related Theory}

Ranging methods focus on estimating the distance between a transmitter and a receiver, by exploiting known signal characteristics. For instance, nodes that are location unaware (free nodes) that are within the communication range (radii) of anchor nodes, can use the RSS from the anchors for distance estimation. If the anchors signal strength is known, along with the attenuation law for signal strength as a function of distance, then the receiver node can use RSS to estimate its distance from the sender [18]. However, in an indoor environment the reception of radio signals becomes more complicated. RSS/distance relationship can vary substantially in an indoor environment due to a host of factors, such as:

- Reflection from smooth surfaces or metallic objects.

- Superposition of electromagnetic fields.

- Diffraction.

- Refraction.

- Diffusion.

- Absorption.

- Polarization of electromagnetic fields.

- Receiver circuit.

In free space, received signal strength is inversely proportional to the square of the distance between two sensors. The free space model provides a measure of path loss as a function of transmitter-receiver (T-R) separation when the transmitter and receiver are within line-of-sight (LOS) range in a free space environment. The model is given by Equation (3) which represents the path loss as a positive quantity in $\mathrm{dB}$.

$$
\begin{gathered}
R S S \propto \frac{1}{(\text { distance })^{2}} \\
\operatorname{PathLoss}(d)=-10 \log _{10}\left[\frac{G_{t} G_{r} \lambda^{2}}{(4 \pi)^{2} d^{2}}\right]
\end{gathered}
$$

where $G_{t}$ and $G_{r}$ are the ratio gains of the transmitting and receiving antennas respectively, $\lambda$ is the wavelength in meters, and $d$ is the Tx-Rx separation in meters. However, the free space path loss equation provides valid results only if the receiving antenna is in the far-field. The far-field is defined as the distance $d_{f}$ given by Equation (4).

$$
d_{f}=\frac{2 D^{2}}{\lambda}
$$

where $D$ is the largest linear dimension of the antenna. For a receiver to be considered in the far-field of the transmitter, it must satisfy $d_{f} \gg D$ and $d_{f} \gg \lambda$. Hence, Equation (3) is not applicable in our situation. Nonetheless, in reality, RSS is highly affected by changes in environmental conditions particularly in an indoor environment. Some of the factors that cause signal strength attenuation make RSS not to correlate with distance, and some of the factors change from 
one enclosed medium to another. Hence, providing a generalized RSS model that could be applicable in all indoor conditions would be very difficult.

RSS or received power, $P_{r}$, is related to distance, $d$ and is given by the log-distance path loss model, which assumes that path loss varies exponentially with distance. The path loss in $\mathrm{dB}$ is given by Equation (5)

$$
\frac{P_{r}}{P_{t}}=G_{t} \cdot G_{r}\left(\frac{\lambda}{4 \pi d}\right)
$$

where, $P_{r}$ is received power, $P_{t}$ is transmitted power, $G_{t}$ and $G_{r}$ are transmitter and receiver antenna gains respectively. $\lambda$ is the wavelength of the signal transmitted and $d$ is distance between antennas. This equation is called the Friis equation. Thus, Equation (6) can be modified as a log-normal shadow model as follows,

$$
P(d)[\mathrm{dBm}]=P\left(d_{0}\right)[\mathrm{dBm}]-10 \gamma \log _{10}\left(\frac{d}{d_{0}}\right)+X_{\sigma}
$$

where $P\left(d_{0}\right)$ represents the transmitting power of an anchor node at a reference distance $d_{0}$ and $d$ the distance between the anchor and the object to be localized, $\gamma$ is the path loss exponent and $X_{\sigma}$ is the shadow fading which follows zero mean Gaussian distribution with $\sigma$ as standard deviation.

Shadowing is due to obstructions caused by hills and buildings, and will be of little effect in our case study, while multipath fading due to the constructive and destructive interference of transmitted signal will be of significance in an indoor environment. Hence, considering that we are interested in indoor localization, particularly in the context of this paper, where we expect the size of a room or hall way to be between $16 \mathrm{~m}^{2}$ and $25 \mathrm{~m}^{2}$, and a maximum distance of not more than $20 \mathrm{~m}$ between the transmitters and receivers, the effect of $X_{\sigma}$ can be neglected. Thus, Equation (7) will be modified as the log-distance path loss model, which assumes that path loss varies exponentially with distance.

$$
P(d)[\mathrm{dBm}]=P\left(d_{0}\right)[\mathrm{dBm}]-10 \gamma \log _{10}\left(\frac{d}{d_{0}}\right)
$$

Consequently, the function of received signal, $f(R S S, \gamma)$ could be estimated. The value of the path loss exponent $\gamma$ varies depending upon the environment. In free space, it is equal to 2 [14].

\section{Experimental Set-Up}

In this section we outlined the methodology applied to obtain the RSS measurements required for RSS-distance estimation. We also describe the location in which the measurements were carried out. One of the main purposes of this research is to propose a reliable non site specific mechanism for estimating RSS relationship with distance. Consequently, the succeeding experiments were conducted to establish that there exists a relationship between RSS and distance that could be explored towards developing an algorithm for the manipulation of 
RSS data in location estimation.

A major problem in indoor localization is mapping RSS to distance, as each indoor environment presents its own unique challenges with regards to the irregularities of RSS with respect to distance. Fashioning a procedure to manipulate RSS values from location aware nodes (anchors) will be of great significance to the effectiveness of the application of WSN in indoor localization. This section describes the testbeds used in conducting the four experiments relevant to our RSS-Distance algorithm. Two of the experiments were carried out using Xbee radios while the other experiment was carried out using Micaz sensor nodes.

\subsection{Experimental Testbed}

In our experiments, we used both Xbee radios and Micaz sensor nodes, both of which use the more advanced CC2420 radio chip and comply with IEEE 802.15.4 WSN standards. CC2420 operates in $2.4 \mathrm{GHz}$ Industrial, Scientific and Medical (ISM) band, with an effective data rate of $256 \mathrm{kbps}$, which is a much higher rate than older radios (see Table 1 ).

In the $2.4 \mathrm{GHz}$ band, the CC2420 has 16 channels, with each channel occupying a $3 \mathrm{MHz}$ bandwidth with a centre frequency separation of $5 \mathrm{MHz}$ for adjacent channels. The radio chip uses an encoding scheme that encodes 32 chips for a symbol of 4 bits. The encoded data is then OQPSK (offset quadrature phase shift keying) modulated. The CC2420 radio chip provides useful information on RSS. Using micaz and Xbee radio (both of which use CC2420 chip), the experiment was conducted in Fraser Noble building at the University of Aberdeen with minimal human interference. Nevertheless, interference was generated by the persons taking readings of the radios from the sensor nodes. The received signal is analyzed with regards to the received signal power levels (RSS in $\mathrm{dBm}$ ). The measurements were taken at various distances.

$$
R S S=10 \log \frac{P\left(d_{0}\right)}{P(d)} \quad[R S S]=\mathrm{dBm}
$$

\subsection{Phase 1 of the Experiment}

As earlier stated, the experiments were conducted in two phases. The first phase was to simply establish that RSS could provide a level of reliability as an indicator for localization. The second phase was conducted to generate a reasonable level of data to enable the modeling of RSS-distance normalization algorithm.

Table 1. Comparison of the CC2420 with RFM TR1000 and CC1000 radios.

\begin{tabular}{ccccc}
\hline Radio & Platforms & Data Rate & Keying & Encoding \\
\hline TR1000 & Mica & $13.3 \mathrm{kbps}$ & ASK & SEC DED \\
CC1000 & Mica2 & $19.2 \mathrm{kbps}$ & BFSK & Manchester \\
CC2420 & $\begin{array}{c}\text { Micaz, Telos, } \\
\text { Intel Mote2 }\end{array}$ & $256 \mathrm{kbps}$ & OQPSK & Spread Spectrum \\
\hline
\end{tabular}




\subsubsection{Xbee Radio}

This experiment was conducted for the purpose of establishing the level of connectivity and reliability of RSS values between transmitter and receiver. Two Xbee radios and two laptops were used in the experiment; one radio was configured using a laptop as the Coordinator and the second was configured as the Router. The X-CTU software tool, which is used for running range test was used to configure the radios. ZigBee protocol standard was used as the protocol for communication between the radios. RSS readings were taken at various distances from the Coordinator to the Router and vice-versa, also, the theoretical received power was calculated for each corresponding distance. Table 2 is the readings taken during the experiment, while Figure 1 is the schematic layout of the setup.

\subsubsection{First Experiment Using Micaz}

The first experiment using Micaz was set-up to analyze variation in connectivity of free sensor node from a single anchor. The anchors and base station (free sensor node) used in the experiment are made by CrossBow. The sensors were MicaZ MTS 420, while the base station was MIB520. The software, MoteView, a graphical user interface (GUI) based on TinyOS, was used to configure the 3 MicaZ sensor nodes used in the experiment. The MIB520, acting as a base station (BS) was connected to a laptop through a USB port, to be able to measure desired data using the MoteView. This set-up forms an XMesh network. The XMesh network requires that a node be programmed with the BS to form a single unit in order to be able to receive data from the three MicaZ nodes. Table 3 indicates the RSS value of each sensor node at particular distances, while Figure 2 is the schematic representation of the experimental setup.

Table 2. RSS values at varied distance.

\begin{tabular}{cccc}
\hline Distance $(\mathrm{m})$ & Theoretical Rx & Coordinator Rx & Router Rx \\
1 & -30.9 & -49 & -50 \\
3.1 & -40.8 & -50 & -50 \\
6.2 & -46 & -53 & -55 \\
9.89 & -50 & -56 & -56 \\
12.99 & -53.3 & -58 & -58 \\
16.09 & -55.2 & -53 & -53 \\
\hline
\end{tabular}

Table 3. RSS values at varied distance using Micaz.

\begin{tabular}{cccc}
\hline Distance $(\mathrm{m})$ & Node 1 $(\mathrm{dBm})$ & Node 2 $(\mathrm{dBm})$ & Node 3 $(\mathrm{dBm})$ \\
\hline 1.35 & -69 & -62 & -75 \\
2.50 & -69 & -66 & -69 \\
3.14 & -76 & -69 & -76 \\
3.25 & -70 & -70 & -78 \\
5.25 & -86 & -79 & -82 \\
\hline
\end{tabular}




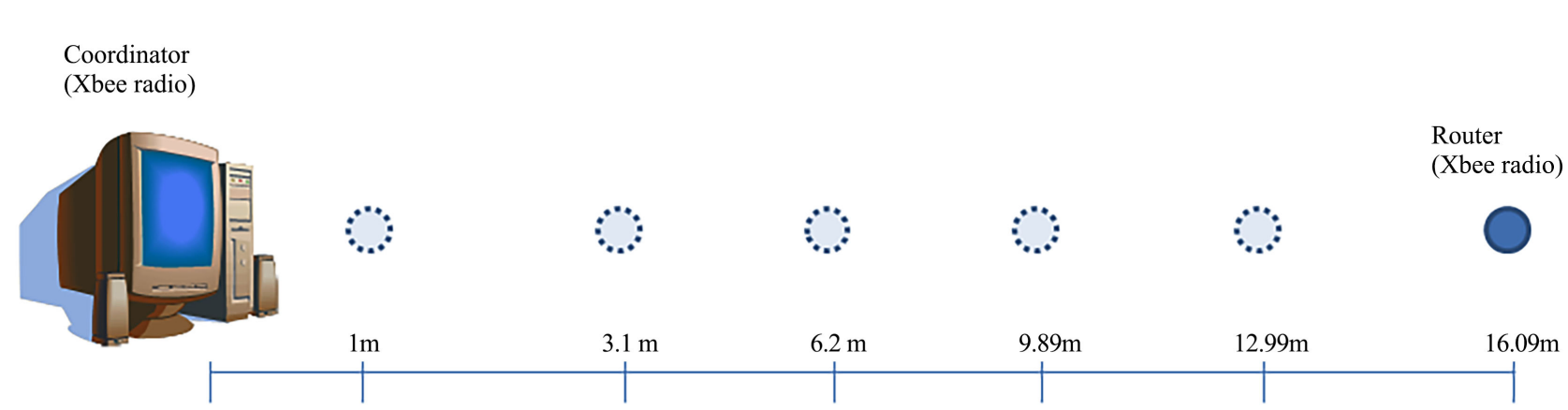

Figure 1. RSS observed from different locations using Xbee radios.

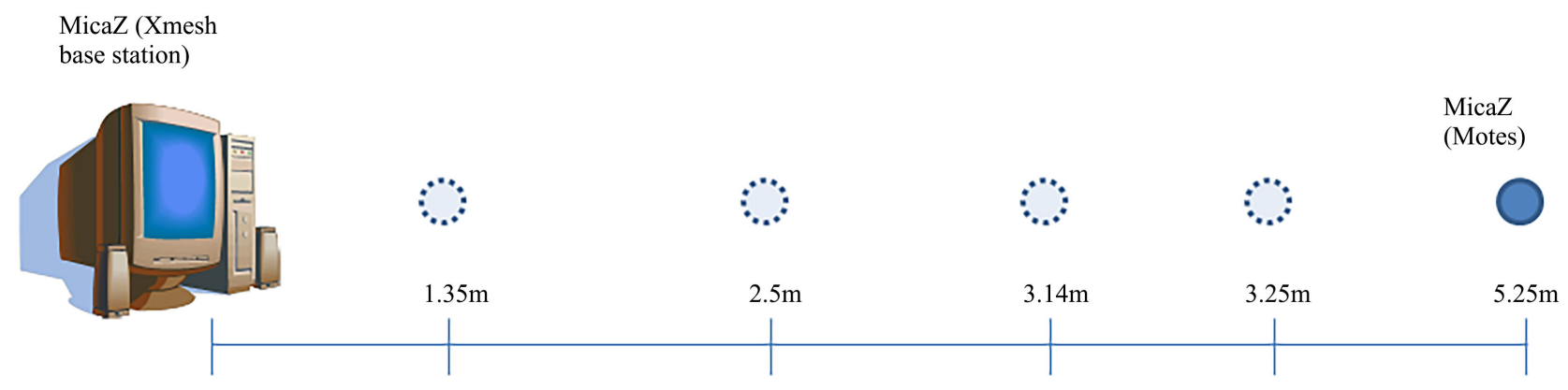

Figure 2. RSS observed from different locations using Micaz sensors.

\subsubsection{Second Experiment Using Micaz}

The second experiment was conducted to analyze the variation in connectivity between a cluster of anchors and a free sensor node. Using 9 MicaZ sensors; one as a base station and the other 8 as two separate clusters, with each cluster comprising four sensor nodes. Each cluster covers a geographical space of about $16 \mathrm{~m}^{2}$ and the cluster about $7 \mathrm{~m}$ apart from each other. The base station was stationed about $2 \mathrm{~m}$ away from one cluster at Location 1 and about $5 \mathrm{~m}$ from the other cluster at Location 2. Fifteen RSS data samples were recorded for each cluster. Using Matlab, the 15 RSS sample readings concurrently taken from 2 different cluster was modeled as a 200 data sample for each cluster. Each data sample was the average reading of the 3 highest RSS values from each cluster. Figure 3 is a schematic layout of the experimental setup.

\subsection{Discussion and Findings for Phase-1 Experiments}

We conducted experiments to investigate the reliability of RSS in localization. Consequently, we set out to measure the RSS values of Xbee radios and MicaZ sensor nodes with respect to distance. The experiments and all measurements were performed in Fraser Noble Building, University of Aberdeen. The Xbee radios and micaz nodes were placed $1.45 \mathrm{~m}$ above the laboratory floor level and the radios were in line of sight. For the two Xbee radios, we transmitted packets at various distances (between 0 and $17 \mathrm{~m}$ ), and collected RSS values of the two radios at various distances using the $\mathrm{X}$-CTU software. Least-square regression analysis was carried out on the data and the regression equation is as given below: 


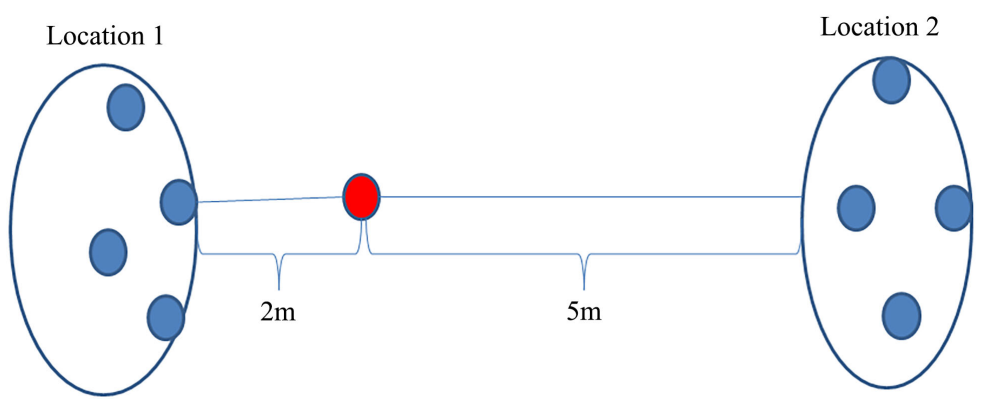

Figure 3. Layout of the sensor nodes.

$$
Y=-13.3-0.626 X_{1}-0.53 X_{2}-0.66 X_{3}
$$

From the least-square regression analysis conducted on the collated data, the adjusted R-square values for the two graphs were reasonably high. The adjusted $\mathrm{R}$-square is an indication of observed variation. Variability in actual radio transmission patterns can have a substantial impact on localization accuracy depending on the localization technique. Unlike the theoretical perfect circles, measured reception distance of radios can vary substantially with environmental conditions and antenna irregularity. However it is documented that proximity based algorithms are substantially unaffected by variation [13]. By implication, from Figure 4, it is safe to conclude that $76.5 \%$ of our observations fit well with the expected linear model, while $23.5 \%$ are because of errors due to multipath fading and the degree of irregularity (DOI) of radio wave pattern. Hence, this degree of accuracy will be sufficient for position estimation using proximity based algorithms.

Also, when the average value of the RSS for the two Xbee radios in the first experiment were used as shown in Figure 5, the adjusted R-squared value rose to $83.45 \%$, which further underscores the reliability of RSS in localization.

Our second experiment was conduct strictly for the purpose of validating our findings from the first experiment with Xbee radios. All the sensors were configured with the MoteView. RSS values as shown in Table 3 were taken at different locations from different distances. Least-square regression analysis was carried out on the data and the regression equation is as given below:

$$
Y=-11.3-0.0309 X_{1}-0.210 X_{2}-0.0319 X_{3}
$$

Figure 6 is the least-square regression plot of the recorded RSS data from the second experiment using Micaz. From the graph it can be seen that the variation is very low (2.9\%). 97.1\% of our observations fits well with the expected linear model. Lastly, Figure 7 shows the plot of RSS measured from the Xbee Coordinator radio to the Router radio and vice-versa, it is near symmetrical, an indication of reliability between transmitted and received signals. 


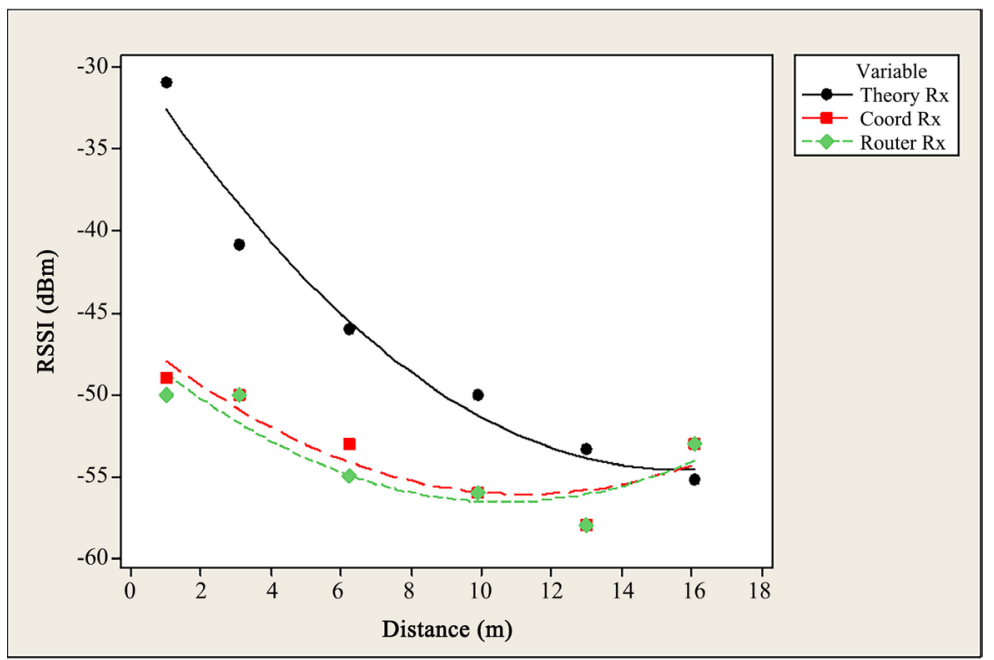

Figure 4. Plot of the Relationship between RSS and distance using Xbee Radio.

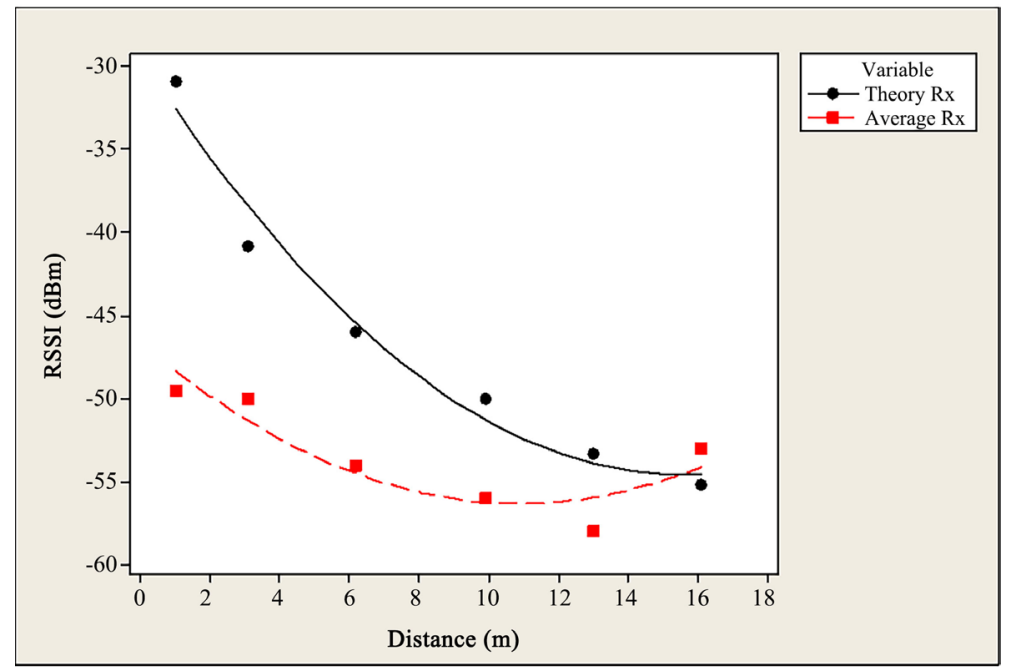

Figure 5. Plot of the Relationship between Average RSS and distance using Xbee Radio.

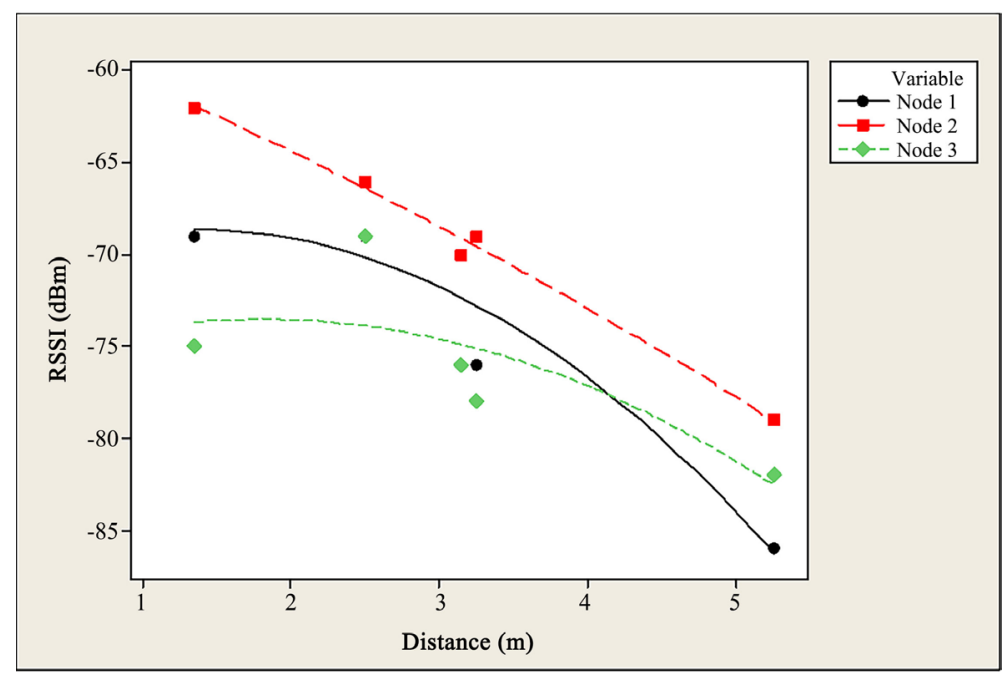

Figure 6. Plot of the Relationship between RSSI and distance using MicaZ. 


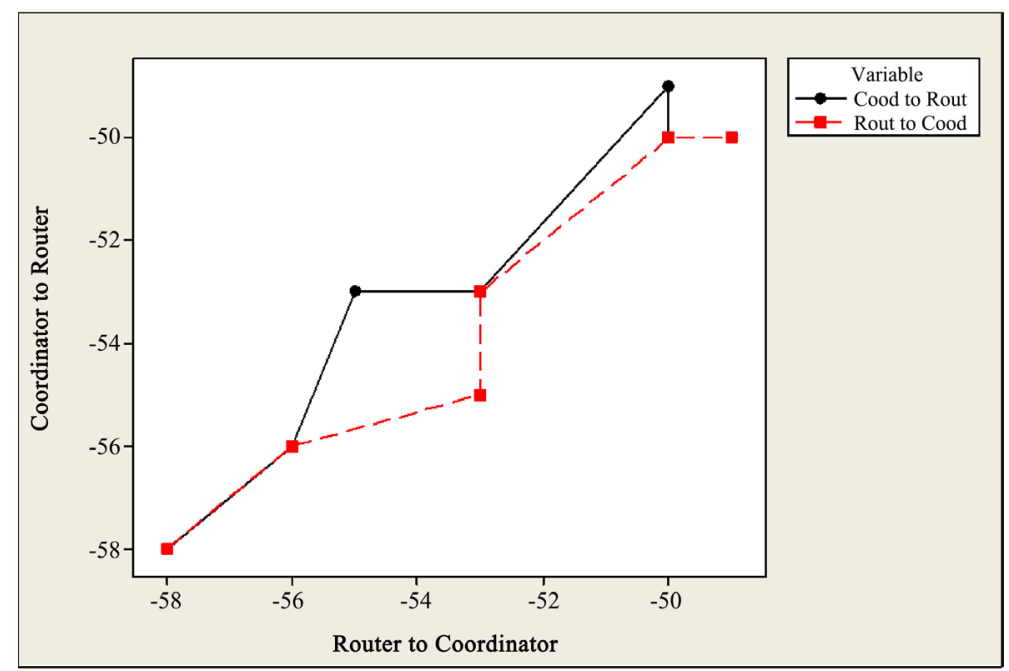

Figure 7. RSSI plot from Coordinator radio to Router radio and vice-versa.

However, it should be noted that there were no physical obstructions between the radios. Nonetheless, the findings in this experiment shows that proximity based algorithms can use RSS as an indicator for localization. The small variations in RSS measured could be attributed to channel variations. This finding is significant, as it shows the positive trend in the level of hardware calibration in the radios employed in WSN. It further buttress the fact that with better calibration, RSS can be used as a key location estimator in localization.

In the second experiment using Micaz, an analysis of the plot shows that the deviation of the cluster of anchors closer to the reference sensor was lower than the deviation of the cluster of anchors farther from the reference sensor (2.317 and 4.795 for the closer and farther clusters respectively). Figure 8 is the plot of the RSS values at the different locations, Figure 9 is a scatter plot indicating the density of RSS reading at each location.

In addition, the range between the maximum and minimum RSS values between the clusters closer to the reference sensor was $7 \mathrm{dBm}$ as compared with 16 $\mathrm{dBm}$ for the clusters farther away from reference sensor. These values should be expected because range estimation error, when it can be quantified, is typically proportional to range such that short range measurements are more accurate within a few meters than longer range measurements. RSS measurement for ranging is a near versus far technique that can provide fairly accurate information about proximity but less accurate with regards to true range. This connectivity variation forms the basis of our algorithm. A knowledge of precise distance is not essential using connectivity, what is important is that the algorithm provides information about the relative positions of objects of interest within a known enclosed geographical space.

\section{RSS-Distance Algorithm Development}

The objective of the algorithm is to establish a best fit relationship between RSS and distance. The aim of our proposed method is to smooth out the irregularities, 


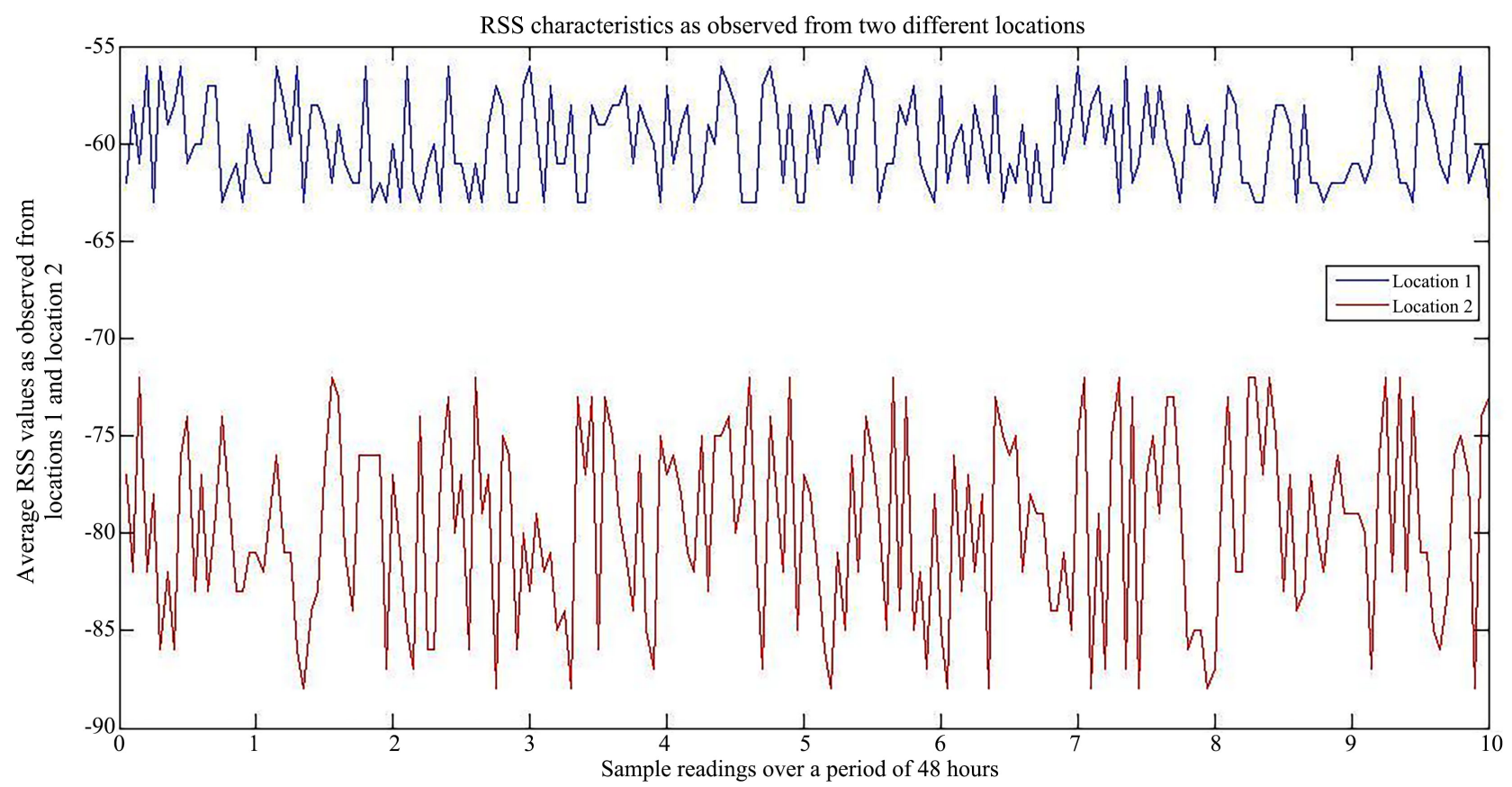

Figure 8. RSS observed from 2 different locations.

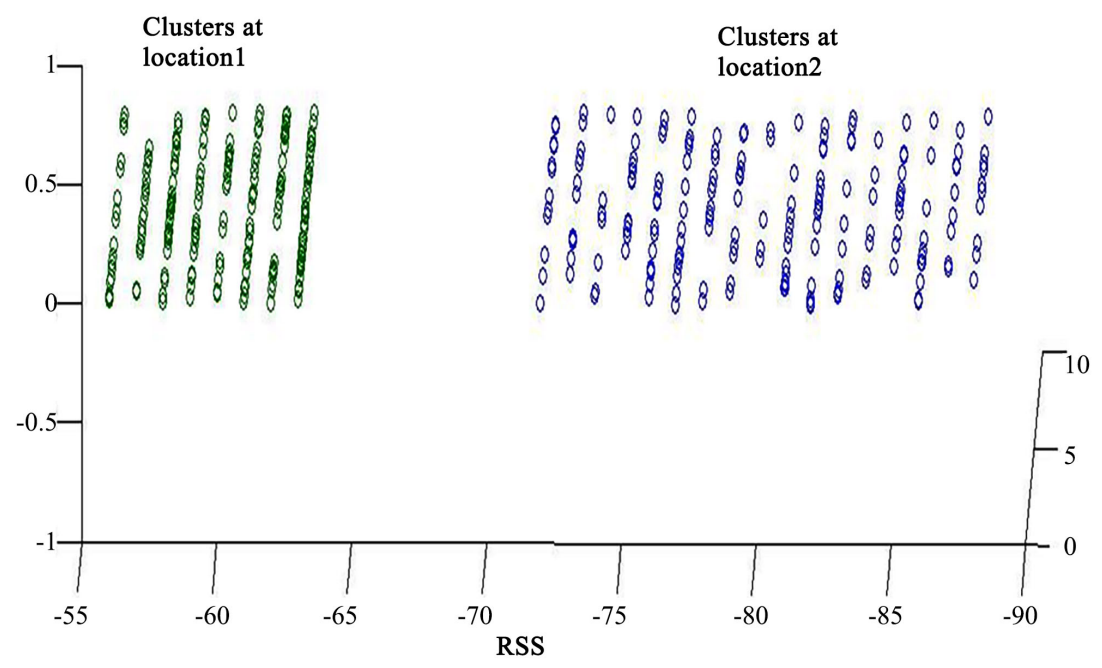

Figure 9. Scattered plot indicating RSS density at each location.

due to multipath effect in the link quality as indicated by the RSS. The idea is to determine a best fit RSS-distance value over a pre-determined window size. In our case study, each sampled average is over a window size of three consecutive RSS values that are between $0.5 \mathrm{~m}$ and $1 \mathrm{~m}$ distance apart.

There is a direct correlation between the error margins in localization and the distance intervals selected for RSS sampling [16]. For higher granularity, the sampled distance interval will have to be smaller, hence, the sample rate over an area will increase. Consequently, the reliability and accuracy of the processed RSS data will increase. However, this will lead to higher demands on the WSN processors and power systems. 


\subsection{Phase 2-Experiment Using Xbee Radios}

This experiment was conducted to collate RSS data over given distances in an enclosed environment, given that it was established from the two preceding experiments that RSS was a good connectivity indicator. Two Xbee radios were configured for this experiment. Using the X-CTU software, one radio was configured as the Coordinator while the other was the Router. The Coordinator served as the anchor radio while the Router was employed as the radio of the free node to be localized. The radios were operated at $2.4 \mathrm{GHz}$ and at full power. The transmission power of the radio was $1 \mathrm{~mW}(0 \mathrm{dBm})$, with a receiver sensitivity of $-92 \mathrm{dBm}$.

The radios were placed on a flat surface $1.2 \mathrm{~m}$ above ground level. The hall way used for the experiment was unevenly shaped with different objects and equipment placed along the hall way. The sides walls were made of a combination of materials such as, bricks, wooden surfaces and metals. RSS readings were taken at $0.5 \mathrm{~m}$ intervals over a distance of $12 \mathrm{~m}$. Table 4 is the collated RSS values.

Xbee radios have an estimated indoor transmission range of $30 \mathrm{~m}$ with a receiver sensitivity of $-92 \mathrm{dBm}$. Signal readings were taken between $0.5 \mathrm{~m}$ and 12 $\mathrm{m}$ at intervals of $0.5 \mathrm{~m}$, which are certainly within the transmission range of the radios. The choice of distance limitation for the experiment was to ensure that RSS readings were consistent and repeatable within the same experimental set-up. Hence, the RSS values in Table 4 are the average sample readings taken over a period of 2 weeks during different periods of the day. The choice of $0.5 \mathrm{~m}$ interval for RSS data collection is indicative of the required level of accuracy for localization. A higher level of accuracy would require a smaller distance sample interval. From Equation (7), we can now model the relationship between distance and RSS as:

$$
Y=20 \log _{10} X-39
$$

where $\gamma=2$ in free space and $\frac{d}{d_{0}}=X$. Based on the data collected, we can express distance as $X(\mathrm{~m})$ and the measured RSS as $Y(\mathrm{dBm})$ for the test bed area. We therefore, set our reference datum using Equation (12), which is the log-distance model for the propagation of the RSS data in free space. We compared the log-distance model with the simple moving average model and our proposed model.

The objective of this method was to check for consistency, which can be deduced by observing the standard deviation of each process from the idealized RSS model. Since the standard deviation in each distance estimate is directly proportional to the increment of the true distance to the transmitter, thus, the accuracy of the resulting location estimate depends directly on the standard deviation [16]. Therefore, we will compare the regression plots of the original data with the ideal log-distance data. The log-distance plots will then be compared with SMA model and our proposed model. 
Table 4. RSS readings at $0.5 \mathrm{~m}$ intervals.

\begin{tabular}{|c|c|}
\hline XY Coordinates (m) & $\mathrm{RSS}(\mathrm{dBm})$ \\
\hline $0.5,0$ & -39 \\
\hline $1.0,0$ & -48 \\
\hline $1.5,0$ & -51 \\
\hline $2.0,0$ & -49 \\
\hline $2.5,0$ & -55 \\
\hline $3.0,0$ & -56 \\
\hline $3.5,0$ & -52 \\
\hline $4.0,0$ & -53 \\
\hline $4.5,0$ & -48 \\
\hline $5.0,0$ & -54 \\
\hline $5.5,0$ & -54 \\
\hline $6.0,0$ & -50 \\
\hline $6.5,0$ & -53 \\
\hline $7.0,0$ & -60 \\
\hline $7.5,0$ & -51 \\
\hline $8.0,0$ & -51 \\
\hline $8.5,0$ & -53 \\
\hline $9.0,0$ & -53 \\
\hline $9.5,0$ & -61 \\
\hline $10.0,0$ & -62 \\
\hline $10.5,0$ & -60 \\
\hline $11.0,0$ & -58 \\
\hline $11.5,0$ & -61 \\
\hline $12.0,0$ & -62 \\
\hline
\end{tabular}

\subsection{Ideal Log-Distance Model}

Equation (12) was used to generate the log-distance RSS values from the raw RSS values. Using Matlab, the regression model of the measured data (raw data) and the ideal log-distance path loss is as shown in Figure 10.

As seen from the graph, the combined influence of reflection, diffraction, and scattering causes multipath effects. At some points, the multipath signal components combine at the receiver to form a distorted version of the transmitted waveform. The signals could combine constructively or destructively depending on phase variations of the component signals. The destructive combination of the multipath components results in attenuated received signal at points where RSS is less than the corresponding log-distance value and constructive where the RSS value is greater than the log-distance value. 


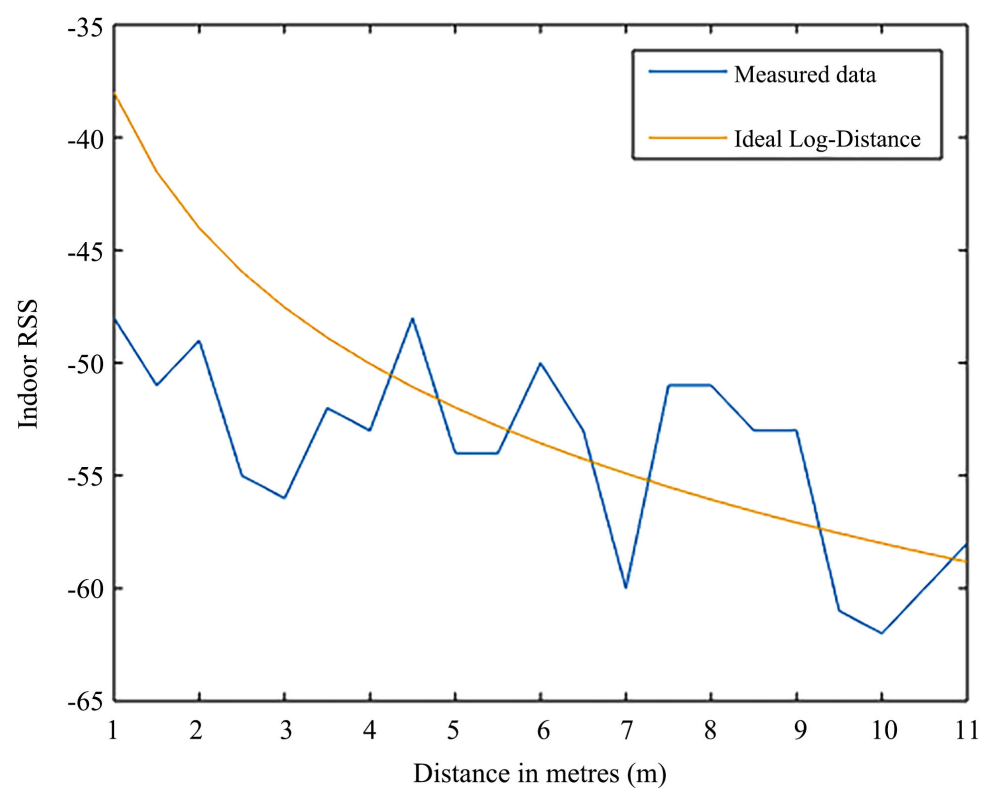

Figure 10. A plot of the measured data and Ideal Log-Distance value.

Two major factors of the plots where selected for analysis; the standard deviation and the range between the minimum and maximum RSS values in each plot. This is as shown in Table 5 .

As expected, propagation of radio signal has a log-normal distribution in free space, hence an idealized RSS value will steadily decrease with distance logarithmically. The log-distance value has a larger range of RSS values than the experimental values. Also, the log-distance plot has a larger standard deviation than the experimental values. There is very little information that could be inferred from this comparison because the idealized log-normal plot pattern is expected to be consistent, while the actual signal plot would change based on environmental conditions. Nonetheless, in this particular plot, the effect of signal amplification of the experimental RSS data resulted in a smaller range between RSS values and this is significant as it influenced a better standard deviation than that of the idealized log-distance model.

\subsection{Simple Moving Average Algorithm}

Simple Moving Average (SMA) is an unweighted mean of a set of data, it is used to identify a trend or direction of a set of data. SMA ensures that variation in the mean are aligned with the variation in the data. The major appeal of the SMA is the ease with which it can be calculated. An advantage to the limited processing and energy power of off the shelf sensor nodes. In our situation, given the set of measured RSS values obtained at the receiver, over a maximum distance of $12 \mathrm{~m}$ from the transmitter at intervals of $0.5 \mathrm{~m}$. This situation is depicted in Figure 11.

For a set of RSS values, the SMA for a group of equally weighted data with a window size of three is; 


$$
R S S_{k}=\frac{a_{k-1}+a_{k}+a_{k+1}}{3}
$$

which can be represent by the Equation (12) below;

$$
R S S_{k}=\frac{1}{3} \sum_{k}^{n-1} a_{k-1}+a_{k}+a_{k+1}
$$

where $n$ and $k$ are positive integers and $n=k+1$.

A regression model of SMA along with the original model of the raw RSS values and the ideal log-distance value is as shown in Figure 12.

Table 6 shows that the RSS range value of the measured data and the SMA are similar in range; 14 and 15 respectively. However, the application of SMA to the measured data improved the standard deviation of the data from 4.206 to 3.62.

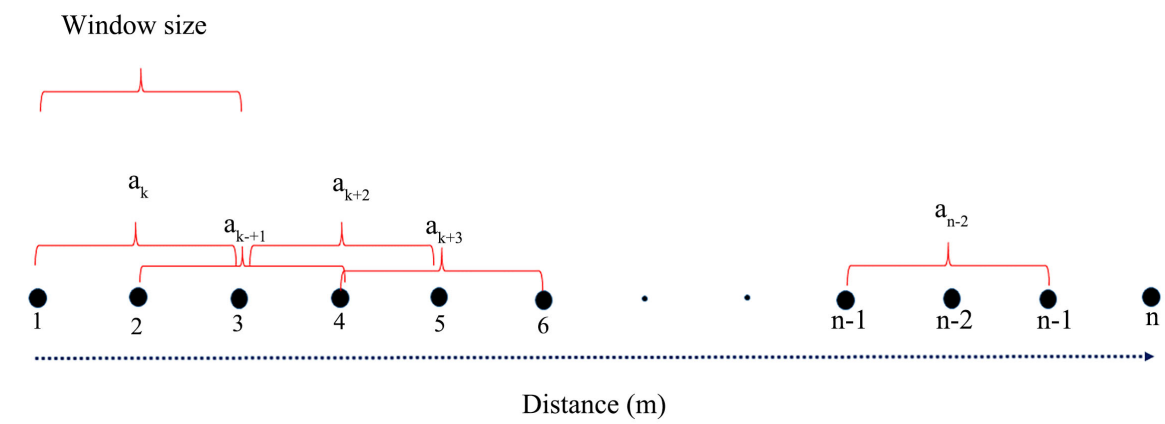

Figure 11. A pictorial representation of SMA estimation process.

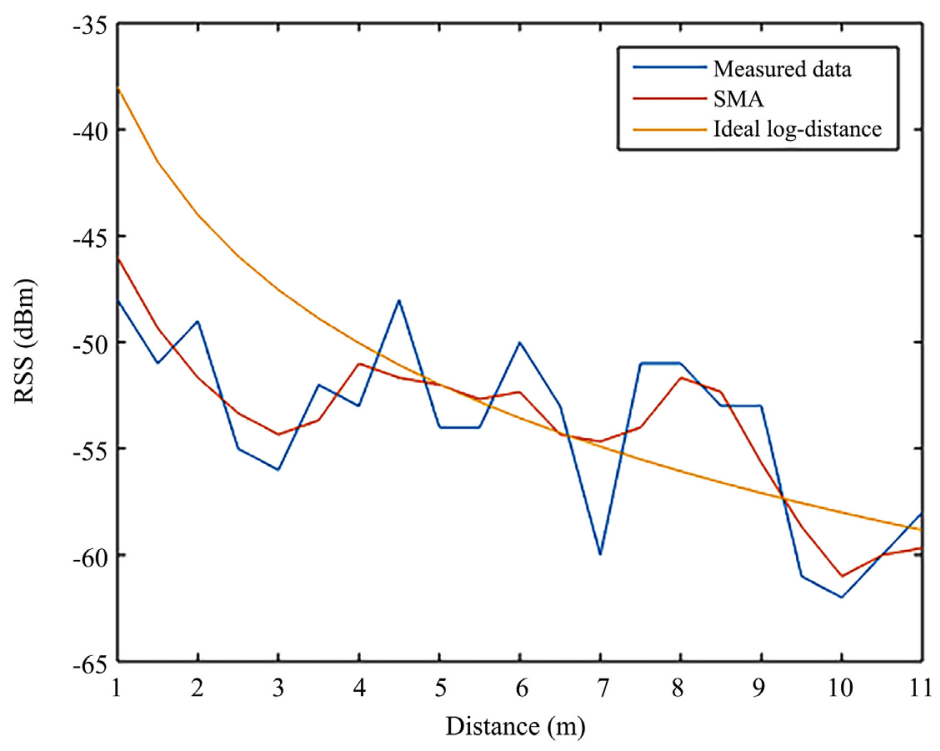

Figure 12. A regression plot of SMA, measured data and the ideal log-distance values.

Table 5. Regression analysis of measured RSS values and idealized model.

\begin{tabular}{ccc}
\hline & Experimental RSS data & Ideal log-distance data \\
\hline STD & 4.206 & 5.862 \\
Range & 14 & 20.83 \\
\hline
\end{tabular}


Table 6. Regression analysis of measured RSS values and SMA.

\begin{tabular}{ccc}
\hline & Experimental RSS data & SMA data \\
\hline STD & 4.206 & 3.62 \\
Range & 14 & 15 \\
\hline
\end{tabular}

\subsection{Proposed Algorithm}

In our experiment, we took measurements at $0.5 \mathrm{~m}$ intervals, which ensured that we were able to compare three discrete signal measurements that were within a $0.5 \mathrm{~m}$ radius. As earlier noted, the major problem with signal measurement in an indoor environment is the consistency in the RSS-distance relationship. Hence, the focus of our proposed algorithm was to enforce a level of consistency. Thus, three discrete and sequentially measured signals will be compared, and the maximum RSS value of the compared RSS data will be selected, this process will be repeated until all the observed data are considered.

In our case, the RSS values at $1.0 \mathrm{~m}, 1.5 \mathrm{~m}$ and $2.0 \mathrm{~m}$ were compared and the maximum of these values selected for the $1.0 \mathrm{~m}$ position. These same steps will be repeated to get a new RSS value for the position $1.5 \mathrm{~m}$, given a set of discretely measured RSS signals taken at a sample interval of $0.5 \mathrm{~m}$;

$$
R S S_{n}=a_{1}, a_{2}, a_{3}, \cdots, a_{n}
$$

Hence, selecting the first three measured RSS data, the new RSS, $X_{1}$ will be;

$$
X_{1}=\left|\max \left\{a_{1}, a_{2}, a_{3}\right\}\right|
$$

likewise;

$$
\begin{gathered}
X_{2}=\left|\max \left\{a_{2}, a_{3}, a_{4}\right\}\right| \\
X_{k}=\left|\max \left\{a_{k}, a_{k+1}, a_{k+2}\right\}\right|
\end{gathered}
$$

where $n$ and $k$ are positive integers; and $k+2 \leq n$. We applied our proposed algorithm to the measured RSS data and applied regression model to compare it with the original RSS data and the ideal log-distance data. This is displayed in Figure 13.

Using our proposed algorithm, Table 7 shows that our proposed algorithm has a higher RSS data range of 21 than the measured RSS value, which is 14 . Likewise, it also has a higher deviation than the measured RSS value. The proposed algorithm has an approximate standard deviation of 4.4 compared with 4.2 for the measured value.

\section{Discussion and Findings}

Indoor conditions make RSS-distance relationship fairly unreliable. The objective of most algorithms is to create a form of consistency between RSS and distance. A correlation between RSS and distance that is very similar in characteristics with the idealize model or propagation of RSS in free space is desired. In this case, we have an idealized situation using the log-distance model. Consequently, the best algorithm as presented in this paper will be the algorithm that best rep- 
licates the characteristics of the log-distance model. Therefore, the key characteristics that will be the focus of our discussion will be range and standard deviation. Using Figure 14 and Table 8 we will examine each of these factors one after the other.

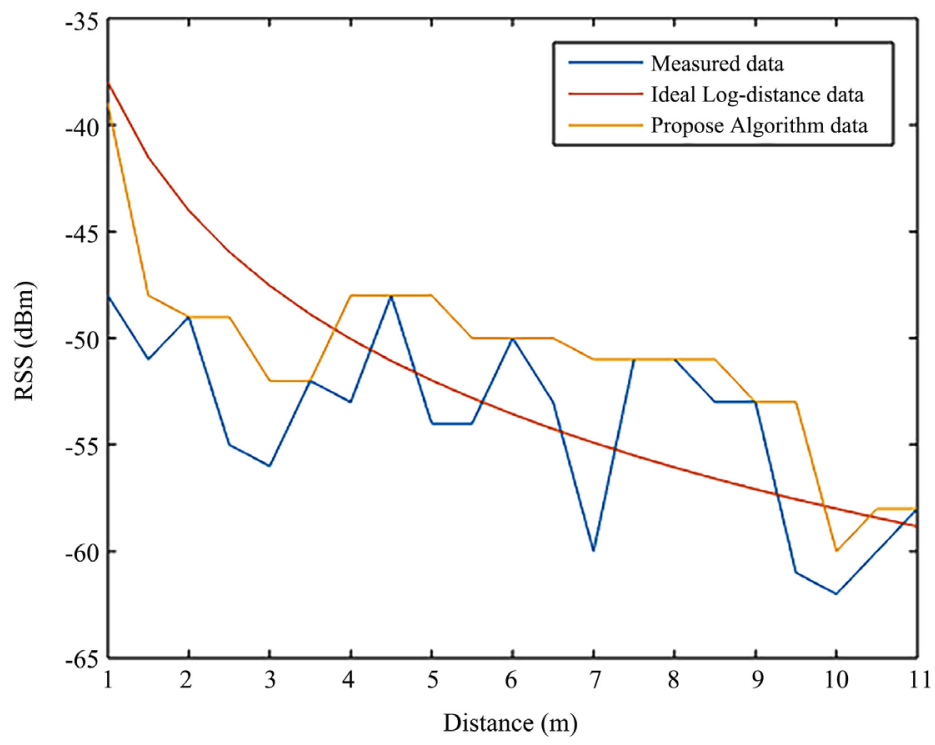

Figure 13. A regression plot using the proposed algorithm, measured data and the ideal log-distance values.

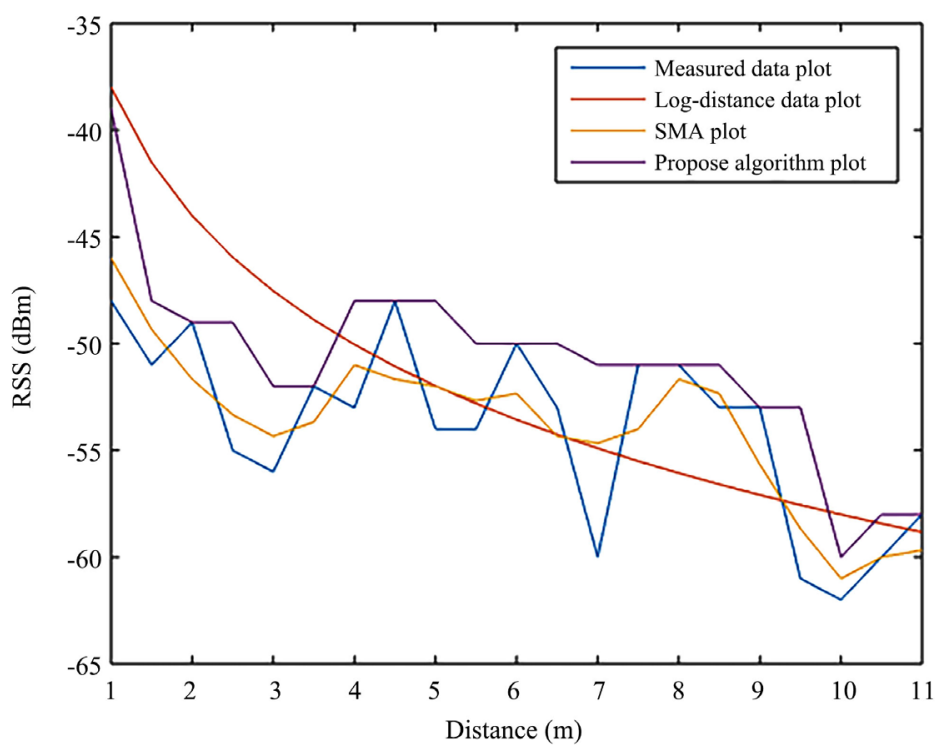

Figure 14. A regression plot of measured data, ideal log-distance values, SMA and the proposed algorithm.

Table 7. Regression analysis of measured RSS values and proposed algorithm values.

\begin{tabular}{ccc}
\hline & Experimental RSS data & Proposed Algorithm data \\
\hline STD & 4.206 & 4.358 \\
Range & 14 & 21 \\
\hline
\end{tabular}


Table 8. Log-distance plot compared with Raw RSS, SMA and proposed algorithm.

\begin{tabular}{ccccc}
\hline & STD & Range & STD Difference & Range Difference \\
\hline Measured data & 4.206 & 14 & 1.656 & 6.83 \\
Log-distance & 5.862 & 20.83 & - & - \\
SMA & 3.62 & 15 & 2.242 & 5.83 \\
Proposed Algorithm & 4.358 & 21 & 1.504 & 0.17 \\
\hline
\end{tabular}

In this paper, range is a measure of the log-normal decay of the RSS values with respect to distance. It will be misleading to use the experimental RSS data as the reference data, as the nature of this data will change based on environmental conditions. What is consistent and expected is a log-normal decay of RSS with increasing distance in free space. Therefore, a good algorithm will seek to modify experimental RSS data to reflect the ideal log-distance model. The algorithm should be close in value to the log-distance data. In our experiment, looking at Table 6, our proposed algorithm best fit this characteristic. Looking at the range values, the proposed algorithm returned the smallest differential (1.504) when compared with the ideal log-distance model.

The second value is the standard deviation. The standard deviation indicated in Table 8 could be misleading, as it is only the deviation of the group of data within each algorithm. To establish a direct relationship between SMA and our proposed algorithm, we processed the standard deviation of the RSS values generated through each process, then subtract the RSS value of each algorithm from the corresponding log-distance value at certain intervals. Through this process, we observed that the proposed algorithm gave the lowest standard deviation differential (0.17). This shows that our proposed algorithm is closer in form to an ideal log-distance model than that of SMA and much better than that of the measured data. Thus, there is a clear indication that our proposed algorithm best replicates the raw RSS values to a form that best fit an ideal log-distance signal model.

\section{Conclusions}

RSS is a free WSN resource for localization, however, with a high level of uncertainty when used in its raw form for indoor distance estimation and consequently, poor localization estimates. Experiments were conducted to establish that RSS was a reliable connectivity index that could be manipulated for location estimation. The main idea behind our algorithm was to select the best fit RSS value within a range of relatively small contiguous set of raw RSS values called a window size. This was to ensure consistency between RSS and distance and also to reduce outlier effects in RSS-distance values. We observed that this approach improved the trend of RSS-distance relationship when compared with an idealized radio wave propagation model. It is believed that the proposed RSS-Distance algorithm when applied to raw RSS data prior to localization will reduce location estimation errors. 
We believe that this novel procedure will improve localization of objects both indoors and outdoors. Nonetheless, we are aware that the proposed algorithm still needs to be subjected to experimental rigors, where RSS is a key determinant factor for location estimation. However, we believe this process will be of significant relevance to proximity based algorithms such as centroid localization algorithm. Furthermore, it is also believed that this algorithm will have strong application towards improving the accuracy of other range-based algorithms with respect to distance estimation, especially range-based algorithms that use Time of Arrival (TOA) for estimation. This possible field of application could form the basis for future research.

\section{Conflicts of Interest}

The author declares no conflicts of interest regarding the publication of this paper.

\section{References}

[1] Mao, G. and Fidan, B. (2009) Introduction to Wireless Sensor Network Localization. Localization Algorithms and Strategies for Wireless Sensor Networks, 1-32. https://doi.org/10.4018/978-1-60566-396-8.ch001

[2] Krach, B. and Robertson, P. (2008) Integration of Foot-Mounted Inertial Sensors into a Bayesian Location Estimation Framework. 2008 5th IEEE Workshop on Positioning, Navigation and Communication (WPNC2008), 55-61.

[3] Priwgharm, R. and Chemtanomwong, P. (2011) A Comparative Study on Indoor Localization Based on RSSI Measurement in Wireless Sensor Network. 2011 Eighth IEEE International Joint Conference on Computer Science and Software Engineering (JCSSE), Nakhon Pathom, 11-13 May 2011, 1-6. https://doi.org/10.1109/JCSSE.2011.5930074

[4] Willoughby, T.R., Kupelian, P.A., Pouliot, J., Shinohara, K., Aubin, M., Roach, M., Skrumeda, L.L., Balter, J.M., Litzenberg, D.W., Hadley, S.W., et al (2006) Target Localization and Real-Time Tracking Using the Calypso 4d Localization System in Patients with Localized Prostate Cancer. International Journal of Radiation Oncology, Biology, Physics, 65, 528-534. https://doi.org/10.1016/j.ijrobp.2006.01.050

[5] Merhi, Z., Nahas, M., Abdul-Nabi, S., Haj-Ali, A. and Bayoumi, M. (2013) RSSI Range Estimation for Indoor Anchor Based Localization for Wireless Sensor Networks. 201325 th IEEE International Conference on Microelectronics (ICM), 1-4.

[6] Parameswaran, A.T., Husain, M.I., Upadhyaya, S., et al (2009) Is RSSI a Reliable Parameter in Sensor Localization Algorithms: An Experimental Study. Field Failure Data Analysis Workshop (F2DAO9), 5.

[7] Ramadurai, V. and Sichitiu, M.L. (2003) Simulation-Based Analysis of a Localization Algorithm for Wireless Ad-Hoc Sensor Networks. Proceedings of the International Conference on Wireless Networks, Las Vegas, NV.

[8] Patwari, N., Hero III, A.O., Perkins, M., Correal, N.S. and O'dea, R.J. (2003) Relative Location Estimation in Wireless Sensor Networks. IEEE Transactions on Signal Processing, 51, 2137-2148.

[9] Barralet, M., Huang, X. and Sharma, D. (2009) Effects of Antenna Polarization on RSSI Based Location Identication. 2009 11 th International Conference on Advanced Communication Technology (ICACT2009), 260-265.

[10] Chen, Y., Pan, Q., Liang, Y. and Hu, Z. (2010) Awcl: Adaptive Weighted Centroid 
Target Localization Algorithm Based on RSSI in WSN. 20103 rd IEEE International Conference on Computer Science and Information Technology (ICCSIT), Chengdu, 9-11 July 2010, 331-336. https://doi.org/10.1109/ICCSIT.2010.5565022

[11] Choi, J.H., Choi, J.K. and Yoo, S.J. (2012) Iterative Path-Loss Exponent Estimation-Based Positioning Scheme in WSNS. 2012 IEEE Fourth International Conference on Ubiquitous and Future Networks (ICUFN), Phuket, 4-6 July 2012, 23-26. https://doi.org/10.1109/ICUFN.2012.6261658

[12] Golestani, A., Petreska, N., Wilfert, D. and Zimmer, C. (2014) Improving the Precision of RSSI-Based Low-Energy Localization Using Path Loss Exponent Estimation. 201411 th IEEE Workshop on Positioning, Navigation and Communication (WPNC), 1-6.

[13] $\mathrm{Hu}, \mathrm{L}$ and Evans, D. (2004) Localization for Mobile Sensor Networks. Proceedings of the 10 th Annual International Conference on Mobile Computing and Networking, Philadelphia, PA, 26 September-1 October 2004, 45-57. https://doi.org/10.1145/1023720.1023726

[14] Oguejiofor, O., Okorogu, V., Adewale, A. and Osuesu, B. (2013) Outdoor Localization System Using RSSI Measurement of Wireless Sensor Network. International Journal of Innovative Technology and Exploring Engineering, 2, 1-6.

[15] Rahman, M.S., Park, Y. and Kim, K.D. (2012) RSS-Based Indoor Localization Algorithm for Wireless Sensor Network Using Generalized Regression Neural Network. Arabian Journal for Science and Engineering, 37, 1043-1053. https://doi.org/10.1007/s13369-012-0218-1

[16] Vander Stoep, J. (2009) Design and Implementation of Reliable Localization Algorithms Using Received Signal Strength. PhD Thesis, University of Washington.

[17] Yun, S., Lee, J., Chung, Y. and Kim, E. (2007) Centroid Localization Method in Wireless Sensor Networks Using Tsk Fuzzy Modeling. ISIS 2007 Proceedings of the 8th Symposium on Advanced Intelligent Systems, 971-974.

[18] Adewumi, O.G., Djouani, K. and Kurien, A.M. (2013) RSSI Based Indoor and Outdoor Distance Estimation for Localization in WSN. 2013 IEEE International Conference on Industrial Technology (ICIT), Cape Town, 25-28 February 2013, 1534-1539. https://doi.org/10.1109/ICIT.2013.6505900 